\title{
ETNOGRAFÍA CONCÉNTRICA Y DIDÁCTICA. NOTAS PARA NO-ANTROPÓLOGOS
}

\author{
Concentric ethnography and didactics \\ Notes to non-anthropologists
}

José Luis Ramos Ramírez ${ }^{1}$

Fecha de recepción: 13 de abril de 2016.

Fecha de aceptación: 19 de Agosto de 2016. 


\section{Resumen}

Ante la necesidad de capacitar a estudiantes y profesionales que no son antropólogos en el quehacer etnográfico se han publicado múltiples manuales que presentan esta labor como exclusivamente metodológica, incluso como un digno ejemplo del enfoque cualitativo; sin embargo al revisar la figura que se oferta en esta literatura encuentro que es una imagen que no hace justicia a la complejidad que significa la Etnografía. Por ello, en este ensayo presento una serie de notas aclaratorias para que se visualicen sus distintos aspectos o caras que muestra la denominada Etnografía. También, incluyo el reto que significa llevar a cabo investigaciones etnográficas en espacios más urbanos y contemporáneos, siendo un ejemplo el caso de la Etnografía Organizacional. Para concluir, anoto algunas recomendaciones, destacando un esquema de trabajo que denomino "Etnografía Concéntrica", dirigidas a los jóvenes investigadores interesados en incursionar en este campo del conocimiento para orientar sus pesquisas respectivas y que no son antropólogos.

Palabras clave: etnografías, Etnografía Organizacional, enfoque, nivel de conocimiento y texto etnográfico.

\section{Abstract}

Taking into account the need to train students and professionals who are not anthropologists in the ethnographic work, multiple manuals have been published presenting this work as purely methodological, even as a well-intentioned example of qualitative approach; however when reviewing the concept offered in this literature I find that is an image that does not do comply with the complexity that Ethnography involves. Therefore, in this essay I display series of clarifications for display various aspects or faces showing the so-called Ethnography notes. Also, I include the challenge of conducting ethnographic research in more urban and contemporary spaces, an example being the case of Organizational Ethnography. To conclude, by adding a number of possible recommendations, highlighting a work framework the way I name it "Concentric Ethnography", designed to young researchers interested in entering this field of knowledge to guide their investigations and who are not actually anthropologists.

Keywords: ethnographies, Ethnography Organizational approach, level of knowledge and ethnographic text. 


\section{Introducción}

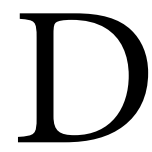

urante el 2015, en cuatro fechas diferentes, llevamos a cabo el Seminario "Introducción a las metodologías etnográficas aplicadas al estudio de las sociedades contemporáneas", para estudiantes de pre y posgrado ${ }^{2}$. Lo peculiar del grupo fue que los asistentes provenían de diferentes carreras a la Antropología, de Medicina, Derecho, Educación, Ingeniería, Literatura, Psicología y Trabajo Social. Condición que volvió altamente interesante la experiencia pedagógica, pero también obligó a revisar y reflexionar sobre la tarea de promover la investigación etnográfica para no-antropólogos.

Al término de esta actividad académica, pude apreciar la necesidad de realizar una labor docente más puntual sobre el quehacer etnográfico, además de fincar una didáctica más apropiada para aquellos interesados en aprenderlo y que no tienen las bases antropológicas que sostienen a la Etnografía. Descubrí que los antecedentes formativos de los asistentes al seminario expresaban una idea esquemática y común de ella, como un método de investigación cualitativa en contraposición a los métodos cuantitativos. Al momento de acercarse a ella, esa fue la versión que les ofrecieron algunos profesionales no-antropólogos.

Son varios los rubros por atender y para este ensayo me interesa compartir diversas notas acerca de la Etnografía buscando acrecentar el interés entre los no-antropólogos, además de aclarar varios puntos que posibiliten un mejor empleo. El contenido del presente texto está orientado en aclarar ciertas ideas un tanto confusas entre los estudiantes no-antropólogos, que están aprendiendo a emplear la Etnografía como un método de investigación cualitativa. A lo largo de la exposición atenderé a dos cuestiones que se desprenden de la frase anterior: en primer término acerca del falso dilema entre los estudios cuali y cuantitativos. El segundo ítem corresponde a la necesidad de formar académica y profesionalmente a investigadores que no han estudiado la carrera de Antropología. Para lo cual ofreceré una concepción heurística y metodológica de la Etnografía, un tipo particular (la Etnografía Organizacional), cerrando con una propuesta didáctica para enseñar y aprender a investigar etnográficamente.

\section{Una imagen confusa de la etnografía}

Un primer problema que pude detectar corresponde a la imagen confusa que recrean en diversos manuales, particularmente los encaminados a ofrecer una exposición sintética de lo que es la Etnografía, con posibilidades e invitación a utilizarla como una alternativa metodológica atractiva. Tomo como referencia el texto de Ballas y Castillo (2008) que es un buen ejemplo de lo que uno encuentra en numerosos textos metodológicos.

Localizo tres cuestiones que van a provocar la presencia de un enfoque confuso: insistir en concebir a la Etnografía de manera exclusiva como método, enfatizar una mirada micro que excluye el sistema sociocultural más amplio e invisibilizar la existencia de la unidad social (colectividad) que soporta su cultura correspondiente. Voy a detenerme un poco en cada aspecto.

2- Se llevó a cabo en las instalaciones del Colegio de Chihuahua, coordinado por Hugo Gaggiotti (UWE), José Luis Ramos R. (ENAH) y Janeth Martínez M. (UACJ) con apoyo financiero de la Academia Británica, como parte del proyecto “"How to study business and organising on permanent movement: new ethnographic practices to study the borderlands". 
Las autoras para ofrecer su idea de Etnografía la han armado mezclando sin mucha precaución referencias de investigadores "cualitativos" y antropólogos, lo cual es una opción viable e interesante; sin embargo, el problema emerge cuando las citas de los antropólogos son empleadas para apuntalar el énfasis y ruta cualitativa del "método etnográfico".

Énfasis que conecta muy fácil con la mirada microscópica que ensalzan varios metodólogos, como las autoras de referencia, quienes destacan con amplitud el ejemplo ofrecido por C. Geertz (1987) sobre el guiño del ojo -como acto cultural- para entender la calificada descripción densa. Parte de la dificultad que ocurre con esta perspectiva microscópica es que implícitamente la ofertan como Etnografía, cuando otros autores (antropólogos) hablan explícitamente de la microetnografía, al concentrarse en un ámbito específico de la realidad sociocultural para su estudio. Claridad que permite sistematizar una estrategia metodológica apropiada para ese rango, un ejemplo lo encontramos cuando Patricia Mena (2000) describe las tareas académicas.

Un segundo problema aparece al momento de confundir niveles de la realidad, como sucede cuando se obvia y excluye al sistema cultural, ocupando su lugar con aspectos microculturales. Al concentrar la mirada buscando captar el significado de pequeñas acciones, el estudioso va alejándose de investigar la cultura de la unidad social completa, compuesta por los diferentes aspectos económicos, políticos, religiosos, estéticos, de parentesco, etc., que podemos encontrar en las primeras etnografías de las sociedades "primitivas".

El sistema cultural o dimensión simbólica de una determinada unidad social (sociedad) sufre un olvido. En la perspectiva microscópica, el detalle minúsculo diluye por completo el horizonte de la estructura o sistema social (sobre la que descansa la cultura).

De esta forma, al desvanecer o esfumar el sistema sociocultural que ampara cualquier detalle cultural genera un problema nodal para comprender lo que es la Etnografía, promoviendo adoptar una imagen poco clara e inadecuada.

\section{Un falso dilema}

Algunos asistentes anunciaron su interés por la Etnografía al considerarla como un buen ejemplo metodológico cualitativo, así se las ofrecieron por primera vez. Con ello refrendaban una idea que está muy presente en diversos manuales de investigación. Al revisar parte de la literatura que refiere a la investigación cualitativa encuentro que efectivamente presentan una versión dicotómica - y en algunos casos como opuesta- con relación a la investigación cuantitativa. Y entregan como ejemplo cualitativo a la Etnografía. Así, los interesados asimilan esta versión esquemática y reducida, distante de lo que para los antropólogos es la Etnografía.

Bajo este horizonte existen numerosos estudiantes que pretenden aprender a investigar enfatizando una primera preocupación: elegir el mejor método -para lograr preparar una buena tesis- entre los cuantitativos versus cualitativos y es cuando localizan a la Etnografía como una opción viable, en esos términos. Sin embargo, muestran escasa preocupación por los antecedentes teóricos que perfilan esta oferta metodológica, dado que los estudiantes sin antecedentes en la Antropología no la tenían. Dejan de lado a la Antropología y por ello ignoran que al interior de esta disciplina, la distinción ( $y$ sobre todo evaluativa) entre lo cuali-cuanti es un falso dilema; por lo tanto, está ausente cualquier acotación en este sentido.

Contexto que me invita a considerar como primer asunto en el presente texto, ofrecer algunos apuntes que permitan apreciar a la Etnografía como algo más que una simple opción metodológica cualitativa. 


\section{Cuatro dimensiones de la etnografía}

La idea central que deseo exponer consiste en señalar que la Etnografía es más que sólo un método. Su carácter metodológico remite únicamente a una de sus cuatro dimensiones, las otras tres apuntan a un nivel de conocimiento, enfoque y producto de ese procedimiento metodológico.

Quizás a mis pares antropológicos les parezca que sólo repito, de manera esquemática, lo que cualquier antropólogo sabe de la Etnografía; sin embargo, insisto, para esta ocasión mi interés principal de diálogo es con otros profesionales no-antropólogos.

Para desarrollar las siguientes notas he combinado las apreciaciones que ofrecen Marcel Mauss (2006) y Rosana Guber (2001 y 2004). En conjunto hablan de cuatro dimensiones, aspectos o caras que muestra la Etnografía, como enfoque, nivel de conocimiento, método y texto. Lo cual nos advierte de lo limitado que implica realizar una lectura exclusivamente metodológica, por parte de los nuevos aprendices, dejando fuera los otros tres aspectos.

\subsection{Enfoque}

La primera faceta tiene un carácter epistemológico, la Etnografía consiste en una alternativa de construcción de conocimiento. Opción basada en la subjetividad del investigador, en tanto sujeto social que entra en interacción con el -también- sujeto de estudio.

A diferencia del requisito que prevalecía, hace algunas décadas, de generar estudios objetivos, evitar la subjetividad, ahora es reconocida como una condición básica. En la actualidad, el interés del investigador por construir conocimiento acerca de la realidad social lo coloca en su interior, pasa a formar parte de ella misma. El estudioso ocupa una posición, juega un rol, apela a sus referencias y preferencias teórico-conceptuales. Al mismo tiempo reconoce que el sujeto de estudio, su interlocutor, también trae consigo determinadas concepciones, experiencias y afectos. Ocurre un diálogo multiforme.

Importa destacar que de esta primera apreciación epistemológica de la Etnografía, van a generarse dos consecuencias metodológicas: una, el investigador requiere situarse en el lugar de vida de los sujetos a investigar por un tiempo prolongado. Y, la segunda, el profesional debe intentar constantemente conocer el punto de vista del sujeto de estudio.

\subsection{Nivel de conocimiento}

Una segunda cara de la Etnografía, refiere a apreciarla como un nivel de conocimiento de situaciones sociales particulares.

Al momento en que fueron delineándose las Ciencias Sociales, a finales del siglo XIX, quedó fincado el interés antropológico por las sociedades "primitivas", demográficamente manejables por el investigador. Esta característica le permitió proponerse perfilar de manera completa las diferentes poblaciones nativas. Impulsar una mirada holística para entender la lógica cultural de estas sociedades. 
Objetivo de conocimiento que indica la necesidad de apreciar los diversos componentes socioculturales (interconectados) de un grupo, para lograr entender la lógica social específica que guardan, labor que no ha sido apreciada en su justa dimensión; es decir, que cuando leo y escucho a estudiantes preocupados por describir con profundo detalle lo que observan a su alrededor, al parecer confunden el dato exhaustivo con tratar de comprender el sentido de las prácticas socioculturales de las personas y colectividades.

Otro anuncio frecuente en los manuales de investigación cualitativa, es la recomendación de armar "descripciones densas". Percibo a los alumnos, que desean buscar con ahínco ir sumando cuantiosos detalles, cuando la acotación de Geertz (1987) destaca la tarea del etnógrafo, que consiste en acercarse al significado otorgado por los sujetos sobre sus prácticas culturales. La invitación establece una pretendida mirada integral, más que un cúmulo de detalles.

\subsection{Método}

El aspecto metodológico es el más referido, pero de manera aislada con relación a las otras facetas de la Etnografía. Como señalé más arriba le han otorgado el papel de ser un ejemplo de los métodos cualitativos, pero como podrán advertir -para este momento- la Etnografía es más que eso.

Ahora traigo a colación la premisa de considerar a la realidad social compuesta por dos dimensiones para su existencia y presencia, una cualitativa y otra cuantitativa. Para intentar acercarnos a ella debemos siempre tener en cuenta que estas dos dimensiones la configuran. Sus combinaciones, complementariedad, ambigüedad, tránsito e incluso ocultamiento, nos presionan a ser muy atentos de no olvidar esta premisa.

Principio que tratan de respetar los diferentes antropólogos; por ello, el dilema de elegir un método sobre otro, no ha sido un buen consejo en la disciplina antropológica. Por eso prefiero retomar las indicaciones epistemológicas de la Etnografía como enfoque, estrategia subjetiva para construir conocimiento. Pautadas por este objetivo, las diversas tareas van mostrando su lógica metodológica.

El etnógrafo requiere aprender a mirar y a escuchar, estar dispuesto en ir asimilando la cultura de la colectividad de estudio y para ello estará un tiempo prolongado conviviendo con las personas, pertenecientes a esa agrupación. De dialogar con ellas, escuchar su voz, descubrir el sentido que le otorgan a su vida en común. Con una constante y permanente reflexión. Experiencia dialógica e interactiva que debe quedar registrada de manera ordenada y sistemática, proveyendo los elementos necesarios para su análisis e interpretación. Por último, se suma la referencia teórico-reflexiva del etnógrafo a la de los sujetos.

\subsection{Texto etnográfico}

Finalmente, el cuarto aspecto corresponde a la tarea de exponer el contenido etnográfico de la pesquisa. Comunicar en un texto lo descrito y su correspondiente comprensión explicativa de la lógica cultural que guarda la colectividad de estudio. En este rubro aparecen contenidos los tres aspectos anteriores, en la lectura uno advierte el proceso y trayectoria de la investigación.

Reconocer las cuatro facetas etnográficas de una Etnografía, posibilita entender que apreciarla simplemente como un método cualitativo es una consideración muy limitada, ella es mucho más que eso. 


\section{Etnografía organizacional}

Durante la primera mitad del siglo pasado la investigación etnográfica tuvo como foco central a las poblaciones "no-occidentales", "tradicionales", etc., que llegó a considerarse como lo distintivo de la Antropología. Afortunadamente esta perspectiva se modificó, ahora entendemos que la mirada antropológica busca comprender la diversidad cultural, lo que implica la posibilidad y necesidad de llevar a cabo pesquisas en todos los espacios sociales posibles. De investigaciones sobre poblaciones étnicamente diferentes se transitó a indagar lo que ocurre en la vida social de las ciudades, las clases medias, los partidos políticos, las iglesias, los centros de recreación, etc.

Anteriormente interesaba comprender las formas de organización étnicas, ahora -además de mantener ese interés- se amplía la perspectiva para inspeccionar y entender tanto tipos como formas de configuración que muestran las distintas organizaciones del mundo moderno, así se perfila la Etnografía Organizacional para estudiar empresas, bancos, fábricas, hospitales, escuelas, clubes deportivos, etc.

Ahora bien, igual que sucedió con otras modalidades de pesquisa etnográfica (etnografía educativa, etnomarketing, etc.), la analogía de empatar a las colectividades "tradicionales" con las expresiones contemporáneas también operó en la Etnografía Organizacional. Aceptar y promover la recomendación de imaginar y percibir a las organizaciones modernas como si fueran similares a las sociedades "primitivas" permitió que los primeros y posteriores estudios, recurran con frecuencia a operar metodológicamente con estrategias análogas.

Varias de las preguntas etnográficas que propone el investigador apelan a cuestiones de aquellas poblaciones, con un mismo objetivo, entender la lógica cultural de esa "microsociedad". El estudioso busca reconocer cuáles son los "rituales" propios de cada espacio laboral de una aerolínea o bien la circulación de bienes simbólicos en una plataforma petrolera o quizás la influencia de las relaciones de parentesco en una cadena de supermercados, etc.

Posibilidades que nos llevan a preguntarnos sobre la metodología de estudio. El perfil que adquiere la denominada Etnografía Organizacional dialoga con dos elementos fundamentales. En primer término, debe contener los cuatro aspectos de la Etnografía en general, anotados previamente. En segundo lugar, el perfil social particular de los espacios específicos de estudio obliga a un uso creativo de algunas técnicas de investigación. En algunos casos las técnicas empleadas, por los etnógrafos clásicos, precisan adaptaciones; en otros, remite a una necesaria invención de instrumentos adecuados a las expresiones culturales contemporáneas de nuevo cuño.

Pero las adaptaciones e invenciones técnicas no deben llevarnos al olvido de una conexión nodal con la teoría. Es decir, cuál es el conocimiento colectivo sobre el objeto de estudio que precede a cualquier nueva pesquisa, que pretendemos iniciar. En nuestro caso, cuando hablamos de organización a qué nos referimos, cuáles son las características generales en que debemos apoyarnos para armar una etnografía. Identifico 3 cuestiones distintivas: a) la organización no es una realidad dada, sino que es construida por los sujetos y el propio investigador, b) construcción que permite manejar analíticamente la flexibilidad y movilidad que notoriamente muestran las diversas organizaciones que deseamos investigar y c) el aspecto clave a detectar en cualquier tipo de organización es su cultura organizacional, compuesta por ideas, normas y valores que dan sentido a las múltiples acciones y objetos, puestos en movimiento para darle existencia a la propia organización. 
Importa no olvidar que la Etnografía (Organizacional, Educativa o de cualquier tipo) siempre seguirá nutriéndose de referencias antropológicas. Por lo cual me interesa, para este ensayo, mencionar algunos puntos clave que son importantes y que conozcan los interesados en emplear la Etnografía para sus investigaciones particulares.

\section{Algunas notas antropológicas}

Un aspecto central de la Antropología o su labor principal es tratar de conocer, comprender la cultura de los diversos colectivos que habitan en el mundo. Acercarse a entender la lógica cultural de las personas y sus colectividades de pertenencia, base sobre la cual la gente se conduce, hace las cosas. El antropólogo en turno requiere de un instrumental heurístico que le permita llegar a comprender los diferentes horizontes culturales. Y para cumplir con esta labor emplea la Etnografía. Si bien la Etnografía ya no es un coto exclusivo de la Antropología, ésta sigue nutriendo de importantes revisiones, acotaciones y modificaciones a su perfil.

Apunté más arriba la importancia central que tiene la liga entre teoría (Antropología) y metodología (Etnografía), por lo que conviene tener presentes algunas cuestiones señaladas por diversos antropólogos contemporáneos, principalmente provenientes de los países "periféricos", distintos a las metrópolis de la Antropología (Estados Unidos de América, Francia e Inglaterra) (Lins y Escobar, 2008).

Una primera consideración es reconocer que la actividad antropológica, como cualquier otra labor científica, es un producto cultural e histórico. Por lo tanto, existen variaciones a lo largo de su trayectoria como del lugar de donde procede. La propia disciplina ocurre en un ir y venir entre lo pretendidamente universal y los particularmente existente. Por ello, varios antropólogos invitan a tener presente que existen antropologías históricas (nacionales) (Lins y Escobar, 2008), sin dejar de lado el horizonte, aunque sea sólo ideal o metafórico de una sola Antropología.

Reconocer este panorama plural permite apreciar la necesidad e importancia del diálogo entre las distintas posturas antropológicas. Se vuelve imperioso el requerimiento de estudios trans-nacionales o globales. Estos diálogos posibilitan no sólo atender a las interrogantes específicas sobre la lógica cultural particular que muestra y contiene la organización que es estudiada, sino también tener presente otro tipo de preguntas: ¿Etnografía para qué y para quién? Interrogantes que son frecuentes entre los antropólogos. Ahora, estimado joven lector (no-antropólogo), te pregunto ¿en tu campo disciplinario y profesional se cuestionan sobre el propio oficio (como indico que ocurre en la Antropología)? ¿Existen momentos de reflexión y revisión sobre los conceptos, los enfoques, el sentido mismo de la especialidad, etc.? Si no acontece, entonces conviene apreciar esta actividad al interior de la Antropología no como una desventaja (falta de solidez o madurez), sino como una característica que posibilita ser más flexible para atender el carácter dinámico y cambiante de la realidad sociocultural.

A la Antropología, en sus inicios, se le confirió la tarea de investigar a las sociedades no-occidentales (al "otro"), buscó entender esas culturas distintas, pero la inspección estaba fuertemente cargada de "etnocentrismo", evaluar la cultura de los otros a partir de la propia (del investigador). Y como expresión de esa mirada está la propia denominación y calificación de los "otros" como sociedades primitivas, tradicionales, etc. (Grimson, 2011). Afortunadamente, surgió una autocrítica a esa postura, dando pie 
a la emergencia del relativismo cultural: valorar las diferentes culturas en sus propios contextos. No hay culturas mejores que otras. Posicionamiento teórico que transitará al plano metodológico, permitiendo perfilar una tarea constante de alerta, para tratar de controlar la valoración sobre las diferentes culturas. Es decir, estas consideraciones llevadas al plano de la Etnografía permitirán discernir su función (¿para qué?). Una respuesta posible es: la Etnografía permite y busca comprender la particularidad cultural humana, asumiendo de manera sistemática y rigurosa el tratar de controlar cualquier tipo de autocentrismo. De esta forma, lo que podría parecer como una flaqueza de la Antropología, al ser examinada constantemente (en comparación a otras profesiones que mantienen sus cánones, sin ser revisados y mucho menos cuestionados de fondo) permite imaginar salidas a los nuevos contextos contemporáneos.

Una segunda consecuencia, al relativizar la mirada y la comprensión de la diversidad cultural deriva hacia la segunda interrogante (¿para quién?). Además del peso marcado por el relativismo, en la actualidad, es notoria la presencia empoderada de las diversas colectividades étnicas. Presencia que se materializa al formar parte de un público informado y que además es beneficiario directo del conocimiento antropológico. Incluso, el camino va más lejos, ahora solicitan una participación directa en la producción y difusión científica, o cuando menos aparecen peticiones de ciertos estudios. A las instituciones tradicionales (museos, universidades, oficinas de gobierno) ahora es menester considerar la presencia de las propias colectividades.

Abanico de posibilidades que tendrán serias y directas consecuencias en los distintos planos de la Etnografía. Por ejemplo, lo hasta aquí expuesto remite directamente al enfoque, transitamos a un tipo de construcción colectiva de conocimiento entre el investigador y el sujeto de estudio (agente y sujeto). Emergen nuevas y diferentes preguntas desde el sujeto-actor de estudio para configurar el nivel de conocimiento. Se amplían y diversifican las necesidades y estrategias metodológicas. Al operar en el proceso de investigación ya no sólo el investigador actúa, ocurre una especie de trabajo colaborativo. Y el público deja de ser sólo la comunidad de antropólogos, para incluir a los "investigados" como lectores de un texto que habla sobre su cultura.

En este contexto, pero en otro plano, conviene seguir impulsando la cautela y objetivo de gran parte de la Antropología, evitar los diferentes tipos de centrismos (etnocentrismo, androcentrismo, adultocentrismo, etc.) en el desarrollo de cualquier pesquisa. Prevención que importa exportar desde la Antropología hacia las otras disciplinas sociales, interesadas en llevar a cabo investigaciones etnográficas.

Condición que vuelve necesario e imperante mantener un diálogo de las diversas disciplinas con la Antropología, para controlar el centrismo que contienen varias de las aseveraciones y afirmaciones, exclusivas de una experiencia social pretendidamente universal, sin tomar la precauciones necesarias que eviten generalizar de inmediato. La importancia de esto apunta a controlar la presencia de juicios valorativos sobre ciertas conductas socioculturales, que den pie para justificar el intentar modificarlas sin antes entender su lógica cultural interna.

\section{Problemas y retos de las "etnografías"}

El principal problema y reto por afrontar es el tránsito de un tipo de Etnografía clásica, tradicional o convencional -término empleado por Knapp (2000)-, a otro de perfil distinto, propio para atender la 
situación sociocultural distinta que corresponde a las características urbanas de las sociedades complejas contemporáneas. Tránsito, adecuación o analogía etnográfica, que requiere sustantivamente de una creatividad etnográfica para configurar posibles salidas novedosas que sean más pertinentes para los diversos escenarios socioculturales.

Otra cuestión corresponde a cierta limitante heurística que contienen numerosas etnografías, tomaron a las sociedades de estudio como entidades aisladas, cuando las conexiones político-administrativas, de mercado, etc., eran importantes para entender ciertos constructos culturales que representaban una respuesta a la presión colonial o estatal.

La decisión metodológica de trabajar en "microsociedades" presumiblemente manejables por el antropólogo, dieron lugar a imaginar que la organización interna era exclusiva. Al momento de querer etnografiar otras realidades (escuelas, hospitales, etc.) fue más claro el problema de determinar los límites de la unidad social por etnografiar. Por ello, una primera tarea consiste en delinear los límites posibles de la unidad social (colectividad) de estudio y las interconexiones que influyen de manera importante parte del sistema sociocultural interno.

Un tercer asunto general corresponde al tipo de etnografía posible y necesaria conforme a las condiciones sociales del estudio. Aun cuando diversas pesquisas surjan de centros de investigación, los tiempos para los productos se han vuelto más insistentes sobre los investigadores. Si resulta que los promotores y beneficiarios de los estudios son agentes políticos, económicos, sociales, etc., habrá mayor premura con el tiempo.

Para el caso de la Etnografía tradicional se requiere de un año de estancia en el lugar y con la gente, como un mínimo temporal, para realizar y cumplir de manera adecuada con la mirada holística. Sin embargo, cuando los promotores y financiadores apresuran el tiempo para tener los resultados, es necesario generar nuevas formas y estilos de llevar a cabo las investigaciones etnográficas. Teniendo muy presente hasta dónde se particulariza, distingue y desintegra el sistema cultural particular.

Algunas alternativas que han surgido son los miniestudios (Knapp, 2000) la microetnografía (Mena,2000), las etnografías específicas (por ejemplo la etnografía educativa, organizacional, etc.), etnografías "parciales" (que ocurren de manera real pero sin denominación alguna; como cuando se describe un salón de clases, etc.) y la etnografía "microscópica" (que siguiendo el ejemplo del "guiño del ojo" analizan un detalle, una acción minúscula: beber cerveza, comprar un par de zapatos, etc.). Geertz (1987) para explicar lo propio de la descripción densa, pone como ejemplo la diferencia que existe entre un acto cultural (guiñar el ojo, que puede significar complicidad, coqueteo, etc.) y otro de carácter exclusivamente físico (parpadear para lubricar el ojo).

\section{Apuntes metodológicos para no-antropólogos}

La mayor parte del contenido expuesto en las páginas precedentes es conocido por los antropólogos, pero mi interés está puesto preferentemente en los investigadores interesados en acercarse a la Etnografía Organizacional y que no son antropólogos, lo cual no es motivo para que otros colegas y principalmente estudiantes de la ciencia antropológica encuentren puntos de reflexión.

Como indiqué al inicio del ensayo, percibo que los estudiosos de otras profesiones en su primer contacto con la Etnografía lo hacen con una clara intención metodológica, por ello quiero ir 
cerrando mi exposición con algunos apuntes metodológicos para el manejo de diferentes tipos de Etnografías (un ejemplo es la Etnografía Organizacional).

El primero, y que es fundamental, tener presente las consideraciones epistemológicas, teóricas, histórica y textuales, que fui atendiendo en los apartados anteriores para apreciar que la Etnografía no es sólo un ejemplo de método cualitativo (es un enfoque, etc). Lo segundo, es querer elegir entre uno y otro método, para los antropólogos es un falso dilema; pensamos que la realidad sociocultural está compuesta por las dos dimensiones (cuali-cuanti) integradas. Por ejemplo, traten de imaginar cómo pueden indagar sólo cuanti o cualitativamente asuntos que tienen que ver, por ejemplo, con la democracia, el consenso, colectividad, grupos, estratificación, hábitos, etc., cuando los conceptos mismos están apuntando a realidades bidimensionales.

El sentido que tiene observar y registrar múltiples acontecimientos es justo para comprender el sentido que guarda cada uno en relación a los demás; es decir, comprender la lógica cultural que expresan los sujetos de estudio. La descripción densa no equivale a un sinfín de detalles sino la búsqueda de significado de cada evento en un sistema significativo. Estaríamos ante la necesidad e interés de comprender y explicar la cultura de una determinada colectividad.

Al operar metodológicamente la descripción y registros etnográficos, a través del trabajo de campo, tener presente que estas labores están fincadas en determinadas consideraciones epistemológicas y teóricas. Además de ser una metodología, es una opción para construir conocimiento, siendo la herramienta nodal para esta labor la subjetividad del investigador, presente en la interacción social que abre y desarrolla con los sujetos de estudio. Donde el etnógrafo guarda una posición y rol sociales, junto con su propia lógica cultural científica y social.

Entonces el investigador novel no sólo debe aprender a trabajar con la etnografía a nivel metodológico, sino que además requiere saber desenvolverse en las otras tres dimensiones etnográficas.

\section{Conclusiones}

Mencioné al principio del ensayo que un motivo central en los interesados por la Etnografía, cuando se acercan por primera ocasión a ella, es para beneficiarse de sus ventajas metodológicas; posteriormente, aclaré que la Etnografía no sólo es un método. Con esta salvedad ahora puedo concentrarme en esbozar algunas notas, principalmente de su perfil metodológico.

Quiero compartir algunas recomendaciones que intentan ofrecer posibles salidas a la necesidad de aplicar la Etnografía, atendiendo a su caracterización básica y, al mismo tiempo, procurar responder a las nuevas condiciones y realidades socioculturales.

Me interesa ofrecer una alternativa que pueda sumarse a la oferta existente: microetnografía, miniestudios, etc. Propuesta que denomino, provisionalmente, "Etnografía concéntrica" integrada por varios registros "etno-temáticos". Considerando que la Etnografía corresponde a un nivel y tipo de conocimiento sobre un determinado sistema sociocultural particular. Sistema que agrupa diferentes componentes y aspectos socioculturales, los cuales están íntimamente interconectados formando un todo, una totalidad.

Una posibilidad de estudiar esa totalidad es llevando a cabo una Etnografía tradicional, para la cual 
se requiere de un tiempo prolongado (varios años). Otra salida apunta a investigar parcialmente esa totalidad, buscando enfocarse en los distintos componentes e irlos conectando de manera paulatina, hasta lograr armar todo el sistema. De esta forma, cada aspecto podrá irse mostrando a los interesados en un tiempo reducido, pero siempre con la salvedad de que existen conexiones pendientes de ser explicadas para lograr una más adecuada comprensión de la lógica cultural de la colectividad de estudio.

A esta segunda opción la denomino "Etnografía concéntrica", ya que el investigador irá concentrando e integrando los componentes parciales (etno-temáticos) con la mira de configurar un sistema sociocultural. Cada elemento "etno-temático" corresponde a una pesquisa "etnográfica" delimitada, que puede ser atendida en una temporalidad reducida. Una preocupación de los diferentes financiadores de proyectos de investigación es que si bien reconocen a la Etnografía tradicional como interesante, dudan de su pertinencia para estudios con requerimientos temporales más acotados o inmediatos.

Con esta modalidad contemplo la posibilidad de manejar un tipo de investigación etnográfica parcial y más inmediata, pero sin perder de vista la unidad o totalidad sociocultural. Combinación que operativamente debe reflejarse en el planteamiento de las interrogantes de estudio. Querer describir significativamente una determinada acción o conducta social, tiene sentido en la medida que expresa localmente la cultura de la colectividad. Tener en mente, por ejemplo, que un acto económico (o del tipo que sea: político, religioso, estético, etc.) está contenida una parte de la cultura económica del grupo.

Imaginemos que estamos dentro de la biblioteca de una universidad, en donde nos podemos preguntar: quién y cuáles fueron los criterios para ordenar el mobiliario y los libros que son consultados por los alumnos de esa institución. Quizás un arquitecto o un diseñador de interiores, por su cuenta o con el apoyo de un bibliotecario. Es probable que lo primero a considerar haya sido la cantidad posible de usuarios, a quienes se les ubicó bajo la categoría general de ser estudiantes universitarios. Pensaron en una propuesta y la materializaron. La cuestión es si hubo un diagnóstico previo o si están llevando a cabo una evaluación de la distribución espacial y uso del acervo documental por parte de los alumnos. Y si dentro del estudio consideraron incluir el componente etnográfico. Pensemos que sí. Ahora el asunto es qué tipo de pesquisa particular están empleando.

Si el investigador apela a la Etnografía, siguiendo el modelo de algún manual, como método cualitativo, intentará llevar a cabo una descripción detallada de algunos eventos de los asistentes: qué tiempo están, cómo van vestidos, qué libros consultan, etc. Además, su conducta: cuánto tiempo estudiaron, de manera individual o grupal, conforme a la normatividad institucional. Y, también lo que ocurre fuera de esas prescripciones: los asistentes están conversando sobre la fiesta del sábado o llegan a dormir, escuchar música, etc. Al final, el estudioso cuenta con un amplio número de páginas que contiene información muy detallada. Datos que empleará para preparar un resumen de lo que observó y registró. Experiencia de trabajo muy delimitada y que ocurre con frecuencia al contemplar a la Etnografía únicamente como "método cualitativo".

Otro investigador, además de cumplir con la misma tarea, empleará ese cúmulo de información otorgándole un mayor grado de utilidad al pretender encontrar el significado que le otorgan los propios sujetos a esos actos particulares. Entender la preferencia de estudiar de manera individual o grupal, por ejemplo.

Una tercera posibilidad es aplicar la "Etnografía concéntrica" (que estoy proponiendo) para indagar acerca de la conexión entre lógicas culturales; por un lado, la de los especialistas que decidieron montar 
un determinado modelo bibliotecario y, por otro, la de los usuarios que expresan una manera particular de desenvolverse dentro de la biblioteca. Lógicas culturales que están inscritas y reconocidas, en un segundo nivel, como cultura estudiantil, universitaria y escolar. El interés va desplegándose conforme el etnógrafo avanza en sus tareas de descubrir, describir, analizar para explicar y comprender la funcionalidad adecuada o no de la biblioteca, amparada en un sistema de ideas, normas y valores que comparten los profesionales y alumnos de una universidad. Todo ello contenido en la cotidianidad del tiempo y espacio bibliotecario.

Parcialidades (en conocimiento y tiempo) que permitirán ir transitando hacia el sistema sociocultural de esa universidad. En el tipo y uso de la biblioteca estarán contenidos parte de los valores de la institución universitaria que la soporta, materializados en los actos individualizados de estudiar, comer y/o dormir dentro de la biblioteca.

Querer emplear la Etnografía para investigar es porque uno desea, en principio, informarse de lo que las personas y grupos realmente hacen y piensan. Y, en un segundo momento, explicar y comprender por qué lo hacen y piensan de una determinada manera, particularmente el significado que tienen las cosas, muy diferente al que le otorga el especialista. En la mayoría de las profesiones (Administración, Pedagogía, Trabajo Social, Diseño, etc.) los especialistas no toman en cuenta ello, suponen e imaginan lo que piensan y hacen los futuros usuarios, beneficiarios, clientes o del tipo que sea, provocando que con frecuencia ocurran desajustes entre la oferta profesional y las expectativas sociales y públicas. Investigar etnográficamente es una buena opción para reestablecer una buena comunicación entre los especialistas y la gente (usuarios, etc.).

\section{Referencias}

Ameigeiras, Aldo. 2016. El abordaje etnográfico en la investigación social. En Estrategias de investigación cualitativa. Barcelona: Gedisa.

Ballas, Claudia, y Helena Castillo. 2008. Introducción a la etnografía y campos de aplicación en educación. En Etnografía. Campos de aplicación en educación y etapas en el proceso de investigación. Bogotá: Pontificia Universidad Javeriana, 7-20.

Cruces, Francisco y otros. 2003. ¿Confianza, cosmética o sospecha? Una etnografía multisituada de las relaciones entre instituciones y usuarios en seis sistemas expertos en España. Alteridades, 13(25): 77-90. Geertz, Clifford. 1987. La interpretación de las culturas. México: Gedisa.

Grimson, Alejandro y otros. 2011. Descentramientos teóricos. Introducción. En Antropología ahora. Debates sobre la alteridad, compilado por Alejandro Grimson, Silvina Merenson y Gabriel Noel. Argentina: Siglo XXI, 9-31.

Guber, Rosana. 2004. El salvaje metropolitano. Reconstrucción del conocimiento social en el trabajo de campo. Buenos Aires: Paidós.

Guber, Rosana. 2001. La etnografía. Método, campo y reflexividad. Argentina: Siglo XXI.

Knapp, Michael. 2000. Contribuciones etnográficas a la investigación evaluativa. Evaluación del programa de Escuelas experimentales y algunas alternativas. En Métodos cualitativos y cuantitativos en investigación evaluativa, compilado por T.D. Cook y Ch. S. Reichardt. Madrid: Morata.

Lins Ribeiro, y Arturo Escobar. 2008. Antropologías del mundo: transformaciones disciplinarias dentro de 
- Revista de Ciencias Sociales y Humanidades. ISSN-P: 0188-9834 ISSN-E: 2395-8669.

sistemas de poder. México: CIESAS/ENVIÓN/The Wenner-Gren Foundation.

Mauss, Marcel. 2006. Manual de etnografía. México: Fondo de Cultura Económica.

Mena, Patricia. 2000. Esbozo de la metodología del microanálisis del aula bilingüe. En Identidad, lengua-

je y enseñanza en escuelas bilingües indígenas de Oaxaca, compilado por Patricia Mena, Héctor Muñoz y Arturo Ruiz. México: Universidad Pedagógica Nacional, 109-156.

Milmaniene, Magalí Paula. 2013. Desafíos éticos en la investigación social. En La ética de la investigación en ciencias sociales. Interrogantes, debates y desafíos actuales. Buenos Aires: Biblos/Investigaciones y ensayos.

Roca, Jordi. 2001. ¿Antropólogos en la empresa?: a propósito de la (mal) llamada cultura de empresa. Etnográfica, 5 (1): 69-99.

Spedding, Alison. 2013. Metodologías cualitativas: Ingreso al trabajo de campo y recolección de datos. En Pautas metodológicas para Investigaciones Cualitativas y Cuantitativas en ciencias sociales y humanas, coordinado por Mario Yapu. La Paz (Bolivia): U-PIEB, 117-195. 